\title{
DIVERSIDAD DE MURCIÉLAGOS EN BOSQUES DE COLINA DEL RÍO ITAYA, LORETO, PERÚ
}

\author{
María Claudia RAMOS RODRÍGUEZ ${ }^{1,5}$, Sheyla Cristina CEVILLANO PATOW ${ }^{1,5}$, Rolando Marino \\ AQUINO YARIHUAMAN ${ }^{2}$, Ricardo ZÁRATE GÓMEZ ${ }^{3}$, Emérita Rosabel TIRADO HERRERA ${ }^{4}$
}

1 Servicios Ambientales para el Desarrollo Sostenible (SADES PERÚ S.R.L); Calle Santa Rosa N 546, Iquitos, Perú. mitza71@gmail.com

2 Universidad Nacional Mayor de San Marcos. Facultad de Ciencias Biológicas, Lima, Perú. P. 0. Box 575 Iquitos, Perú

3 Instituto de Investigaciones de la Amazonía Peruana. Programa de Investigación en Cambio Climático Desarrollo Territorial y Ambiente (PROTERRA); Av. Quiñones km 2.5, San Juan Bautista, Maynas, Loreto, Perú.

4 Universidad Nacional de la Amazonia Peruana, Facultad de Ciencias Biológicas; Calle Pebas cuadra 5, Iquitos, Perú.

5 Centro Amazónico de Educación Ambiental e Investigación (ACEER); Av. Cusco s/n, Puerto Maldonado, Perú. Iquitos, Perú.

\section{RESUMEN}

Los murciélagos son uno de los grupos de mamíferos más diversos del Perú, también son los más temidos por la población humana que en su mayoría desconoce sus cualidades y potencial ecológico regido por sus estrategias alimentarias. Por ello, con el objetivo de conocer la diversidad de estos mamíferos voladores en bosques de colina de la cuenca alta del río Itaya, del departamento de Loreto, Perú. Entre el 2009 y 2011 se realizó capturas a nivel de sotobosque y medio dosel en 50 unidades de muestreo. El resultado del esfuerzo de 1800 horas/red logró registrar una riqueza $\left({ }^{0} \mathrm{D}\right)$ de 47 especies, equivalente al $79.6 \%$ del total estimado para esta parte de la Amazonía peruana. La ${ }^{0} \mathrm{D}$ muestra diferencias significativas a nivel de estrato $(t=3.28$, $\mathrm{p}=0.01$ ), con mayor cantidad de especies en sotobosque respecto al medio dosel. Además, se incrementó la riqueza de especies con 17 nuevos registros para la zona. Las especies frugívoras Rhinophylla pumilio, Artibeus obscurus y Carollia brevicauda fueron dominantes $(\lambda=0.94)$. Asimismo, las comparaciones proporcionales realizadas en cuanto a tamaño, edad y gremio alimentario, evidenciaron diferencias significativas, aun cuando se mantuvieron homogéneas a nivel de estratos $(<0.0001)$. La diversidad verdadera $\left({ }^{1} \mathrm{D}\right)$ muestra una efectividad de 24.51 especies para los bosques de colina, manteniéndose similar en ambos estratos $(t=0.94, \mathrm{p}=0.24)$. La diversidad de murciélagos registrada en este estudio, podría verse influenciada por el incremento de actividades extractivas durante la última década. Consideramos oportuno tomar medidas de influencia política para gestionar de manera sostenible el uso de los recursos aprovechados en esta parte de la Amazonía Peruana.

PALABRAS CLAVES: Dominancia de murciélagos, Especies efectivas, Estrato de bosque, Gremio alimentario, Quirópteros.

\section{BATS DIVERSITY IN HILL FORESTS OF THE ITAYA RIVER, LORETO, PERU}

\begin{abstract}
Bats are one of the most diverse groups of mammals in Peru, and they are also the most feared by the human population, which mostly ignores the qualities and ecological potential governed by their feeding strategies. Therefore, we had the objective of knowing the diversity of these flying mammals in the hill forests of the upper Itaya river basin, in the department of Loreto, Peru. Between 2009 and 2011, we took captures at the understory and middle canopy level in 50 sampling units. The result of the effort of 1800 hours/net registered a species richness $\left({ }^{0} \mathrm{D}\right)$ of 47 species, equivalent to $79.6 \%$ of the total estimated for this part of the Peruvian Amazon. The ${ }^{0} \mathrm{D}$ shows significant differences at the stratum level $(\mathrm{t}=3.28, \mathrm{p}=0.01)$, with a greater number of understory species than the middle canopy. In addition, species richness was increased with 17 new records for the area. The frugivorous species Rhinophylla pumilio, Artibeus obscurus and Carollia brevicauda were dominant $(\lambda=0.94)$. Likewise, the proportional comparisons made in terms of size, age and food guild, showed significant differences, even when they remained homogeneous at the level of strata $(<0.0001)$. The true diversity $\left({ }^{1} \mathrm{D}\right)$ shows an effectiveness of 24.51 species for the hill forests, remaining similar in both strata $(t=0.94, p=0.24)$. The diversity of bats recorded in this study could be influenced by the increase in extractive activities during the last decade. We consider it opportune to take measures of political influence to manage in a sustainable way the use of the resources used in this part of the Peruvian Amazon.
\end{abstract}

KEYWORDS: Bats dominance, Chiroptera, Effective species, Food Guild, Forest strata. 


\section{INTRODUCCIÓN}

Los estudios sobre diversidad de especies nos permiten conocer los componentes y realizar comparaciones entre ecosistemas (Maclaurin \& Sterelny, 2008). Los cambios que ocurren en la magnitud de diversidad en un ecosistema se deben a diversos factores, que pueden ser alteraciones naturales o provocadas en la dinámica estructural de comunidades que varía según la estacionalidad, determinantes históricos, cambio climático e impactos de actividades humanas (Moreno et al., 2011). Estos cambios o mantenimientos de magnitudes de diversidad pueden justificar acciones que coadyuven a la protección de ecosistemas, conservación de áreas, elaboración de planes de manejo, entre otras medidas que sean de interés priorizar.

El Perú, representa el tercer país con mayor diversidad de mamíferos en el nuevo mundo, siendo los murciélagos uno de los grupos mejor representados con 182 especies (Velazco, 2017), después de los roedores. Asimismo, el Departamento de Loreto representa el $54.40 \%$ con 99 especies de murciélagos del Perú (Baker et al., 2009; Bravo, 2009; Velazco et al., 2010; Lim et al., 2010; Díaz, 2011; Calderón \& Pacheco, 2012; Fernández \& Torres, 2013; Velazco et al., 2014, 2017); seguido por el Departamento de Ucayali con 86 especies (Quintana et al., 2009) y Madre de Dios con 83 especies (Fernández \& Torres, 2013). Durante la última década, la riqueza de murciélagos en el Perú ha incrementado 17 especies, de estos, el 47.06\% habitan la Amazonía nororiental del Perú. En Loreto, la mayoría de los estudios se realizaron en bosques y cultivos aledaños a la carretera Iquitos Nauta y Reserva Nacional Allpahuayo Mishana (RNAM), en tanto que diversas extensiones de bosques han sido pocas o nada exploradas.

Los bosques de la cuenca alta del río Itaya, a pesar de su cercanía a la ciudad de Iquitos, han sido escasamente estudiados. Esta cuenca se caracteriza por presentar en su mayoría bosques de colina baja (Zárate et al., 2013) que albergan una gran variedad de microhábitats propicios para refugios de murciélagos (Calderón \& Rengifo, 2010). La composición florística presenta diferentes formas de vida (hierbas, lianas, arbustos y árboles) que forman parte de la alimentación de murciélagos frugívoros (Cevillano \& Ramos, 2011); estas características podrían estar influenciadas en la composición de especies del área evaluada.

En esta cuenca se desarrollan diversas actividades antropogénicas como la cacería, extracción de madera, cosecha de hojas de irapay (Lepidocaryum tenue) y frutos silvestres, que cada vez son mayores, impactando de manera negativa a las poblaciones de fauna silvestre por desarrollarse sin ningún criterios de manejo sostenible (Aquino et al., 2009), siendo los quirópteros uno de los grupos más afectados.

Los escasos estudios y la creciente actividad antropogénica nos motivaron a evaluar la diversidad de murciélagos en esta cuenca, información que permitirá generar una línea base para monitoreo y ulteriores estudios sobre el estado de conservación de estos bosques, los cuales necesitan urgentemente ser manejados adecuadamente debido a su cercanía a Iquitos y por proporcionar servicios ecosistémicos a este gran ecosistema urbano.

\section{MATERIALES Y MÉTODOS}

\section{ÁREA DEESTUDIO}

El estudio fue conducido en bosques de colina baja (Zárate et al., 2013; MINAM, 2015) de la cuenca alta del río Itaya entre el año 2009 y 2011. Fueron evaluados cuatro sectores: Campamento Calaberitas $\left(-73.77^{\circ},-4.166^{\circ}\right)$, Villa Belén $\left(-73.71^{\circ},-\right.$ $\left.4.29^{\circ}\right)$, Quebrada Yanayacu $\left(-73.71^{\circ},-4.27^{\circ}\right)$, Cabecera Pensión $\left(-73.61^{\circ},-4.40^{\circ}\right)$ con un total de 50 unidades de muestreo ubicados entre los Distritos de San Juan Bautista en la Provincia de Maynas y Distrito de Nauta en la Provincia de Loreto (Figura $1)$.

El área de estudio se caracteriza por presentar un clima moderadamente húmedo y cálido (según clasificación de Thornthwaite). La temperatura media es de $27^{\circ} \mathrm{C}$, variando entre $20^{\circ} \mathrm{C}$ (mínima) y $33^{\circ} \mathrm{C}$ (máxima).

Las precipitaciones totales anuales fluctúan entre $250-300 \mathrm{~mm}$, siendo $130 \mathrm{~mm}$ (Julio) y $370 \mathrm{~mm}$ (marzo) la precipitación mínima y máxima, respectivamente. El régimen hidrológico tiene un comportamiento de crecientes y vaciantes en la selva baja. La humedad relativa media entre los meses de agosto y octubre fue $87 \%$ y entre abril y junio de $90 \%$ (Paredes, 2012).

La vegetación característica del área de estudio corresponden a bosques de colina baja, donde se distinguen asociaciones de formaciones vegetales con abundancia de Lepidocaryum tenue, (localmente conocidos como irapayales) y Duroia sp. y Cordia nodosa (localmente conocidos como: supaychacras).

Entre la vegetación arbórea sobresalen por su mayor predominancia Eschweilera tessmannii, Brosimun acutifolium, Parkia nitida, Pseudolmedia laevigata, Iryanthera elliptica, Virola calophylla, Pouteria guianensis, Abuta imene, Escheweilera coriaceae, Oenocarpus bataua, entre otras (Encarnación, 1993; Zárate et al., 2013). 


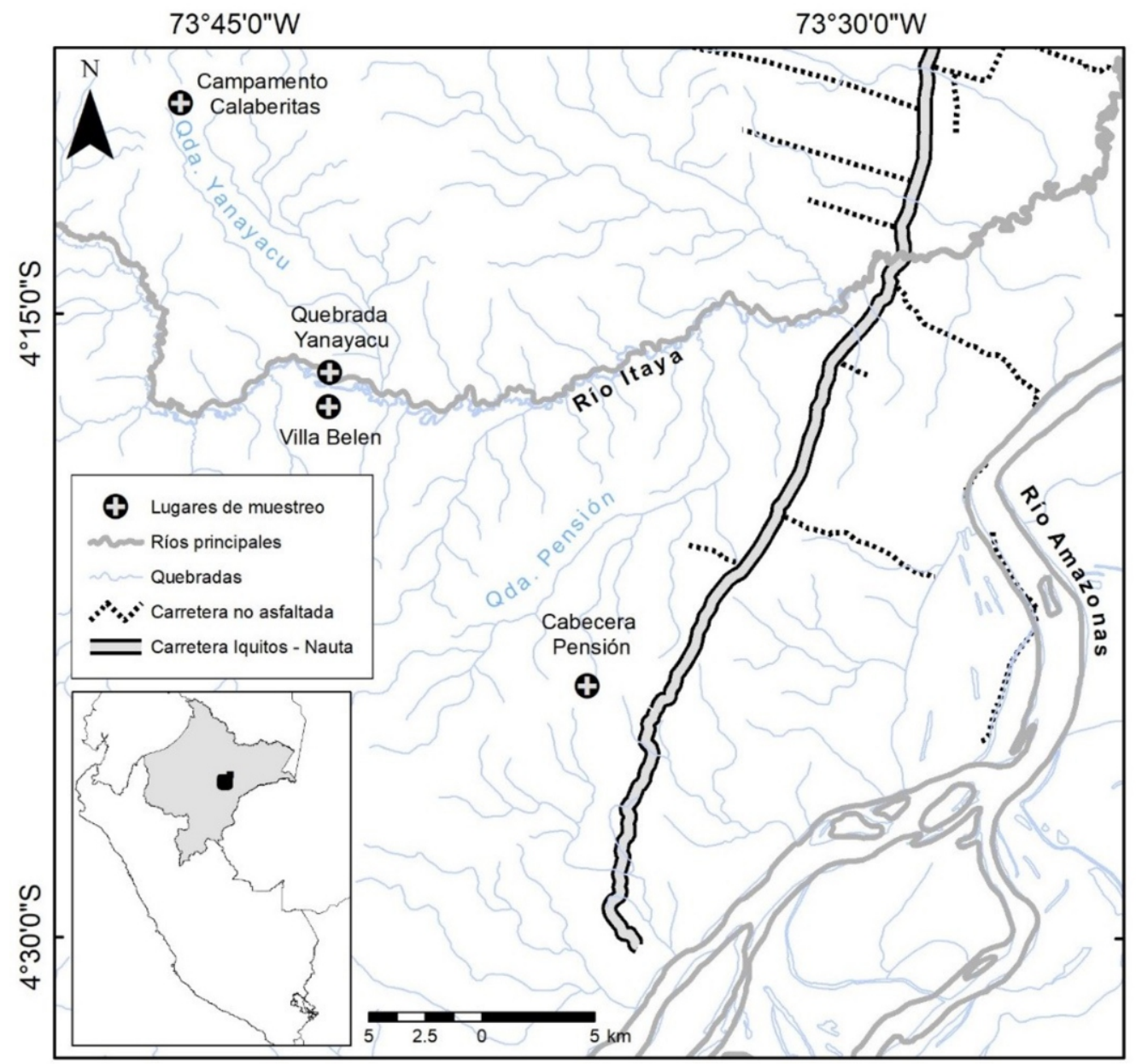

Figura 1. Mapa de la cuenca alta del río Itaya (Perú) mostrando los sitios de muestreo de los murciélagos.

Los bosques de colina evaluados presentan dos gradientes de perturbación: Moderado y Moderado-Alterado, clasificación sustentada en la composición de fauna silvestre y extracción de recursos forestales y no forestales, así como el cambio de uso del suelo (Aquino et al., 2012).

\section{MÉTODOS}

La captura de murciélagos se realizó utilizando redes de neblina (Jones et al., 1996). Entre los meses de Julio a Diciembre de 2009 y Setiembre a Octubre de 2011; durante 50 noches de muestreo se colocaron 300 redes ( 6 redes/noche) con dimensiones de $12 \mathrm{~m}$ de longitud por $2.5 \mathrm{~m}$ de alto y $35 \mathrm{~mm}$ de ojo de malla. Considerando que la diversidad y estructura comunitaria de murciélagos difiere entre los estratos arbóreos (Voss \& Emmons, 1996), se instalaron redes a nivel de estrato vertical: sotobosque $(<5 \mathrm{~m})$ y medio dosel $(5-16 \mathrm{~m})$. Las redes de sotobosque fueron fijadas con "varas" y las de medio dosel con "cuerdas" como un sistema de poleas, las mismas que se mantuvieron activas desde las 18:00 h hasta las 24:00 h y revisadas dependiendo de la frecuencia de captura, entre 30 y $60 \mathrm{~min}$. El esfuerzo de captura (EC) se estandarizó como hora/red, y se obtuvo mediante la fórmula $\mathrm{EC}=\mathrm{N}^{\circ}$ horas $\mathrm{x} \mathrm{N}^{\circ}$ redes (Muñoz, 2001).

Los individuos capturados se colocaron en bolsas blancas de tela y luego trasladados a nuestro campamento base, donde fueron identificados y registrados en una ficha de campo, anotándose datos morfológicos y biométricos. Las especies se identificaron utilizando las claves taxonómicas de Tirira (1999), Simmons (2006) y Gardner (2008). Posteriormente se realizó un registro fotográfico y luego procedimos a liberarlos. El arreglo taxonómico de las especies se hizo con la referencia de Solari \& Martínez-Arias (2014). 
Se consideró las variables de edad, tamaño y gremio alimentario de los murciélagos para realizar comparaciones de las proporciones de individuos entre los estratos de sotobosque y medio dosel. El "tamaño" se clasificó en tres tipos según la longitud del antebrazo: pequeño (menor de $45 \mathrm{~mm}$ ), mediano $(45-65 \mathrm{~mm}$ ) y grande (mayor a $65 \mathrm{~mm}$ ) (Tirira, 1999; Simmons, 2006; Gardner, 2008; Díaz et al., 2011; López -Baucells et al., 2016). En la “edad" se consideró la dentición, pelaje y epífisis de los dedos clasificándolos en cría, juvenil, sub adulto y adulto. El "gremio alimentario" se determinó según las muestras fecales que los murciélagos depositaron en las bolsas de tela y por referencia de Baker et al. (1976).

\section{DISEÑO DE MUESTREO}

Se aplicó un tipo de diseño aleatorio a las unidades de muestreo (sistema de seis redes). Cada una de las redes se instaló de forma sistemática con una separación de 20 a $30 \mathrm{~m}$, intercalando sotobosque y medio dosel, respectivamente. En el sotobosque, las redes fueron instaladas siguiendo las pautas establecidas por Nagorsen \& Peterson (1980) y en medio dosel, a Kunz \& Kurta (1988), modificado por Tirira (1999).

\section{ANÁLISIS DEDATOS}

Los datos obtenidos se analizaron con estadística descriptiva, usando estimadores de medidas de tendencia central y precisión con el programa BioEstat 5.3 (Ayres et al., 2007). La estimación de la riqueza esperada se calculó con los estimadores: Chao1, Chao2, Jacknife1, Jacknife2 y Boopstrap, Michaelis y Mendel, analizados por el programa SDR 4.1.2. (Seaby \& Henderson, 2007).

La diversidad alfa, se analizó en función a la serie de Hill, considerando la Diversidad de Orden $0\left({ }^{\circ} \mathrm{D}\right)$ cuyo valor equivale a la riqueza de especies $\left({ }^{\circ} \mathrm{D}=\mathrm{S}\right)$ y la Diversidad de Orden $1\left({ }^{I} D\right)$, equivalente al número de especies efectivas (Hill, 1973; Moreno et $a l ., 2011)$, obtenidas del exponencial del índice de entropía de Shannon (Jost, 2006; García-Morales et al., 2011), analizados en el programa Past 3.19 (Hammer et al., 2001). La comparación de proporciones de las variables tamaño, edad y gremio alimentario se analizó mediante una Prueba $G$ de homogeneidad en el programa BioEstat 5.3.

\section{RESULTADOS}

En 1800 horas/red se capturó 345 individuos pertenecientes a 47 especies $\left({ }^{0} \mathrm{D}\right)$, agrupados en 26 géneros, 9 subfamilias y 4 familias
(Figura 2, Tabla 1). La familia dominante en los bosques de colina baja fue Phyllostomidae con el 95.65\% de capturas, seguido por Emballonuridae (3.19\%), Vespertilionidae $(0.87 \%)$ y Thyropteridae $(0.29 \%)$. Las especies con mayor éxito de captura fueron los frugívoros Rhinophylla pumilio $(0.49$ ind./horas/red), Artibeus obscurus (0.34 ind./horas/red) y Carollia brevicauda (0.28 ind./horas/red); y los registros únicos fueron Carollia benkeithi, Dermanura anderseni, Desmodus rotundus, Glossophaga commissarisi, Lophostoma carrikeri, Myotis riparius, Peropteryx macrotis, Thyroptera tricolor, Vampyressa aff. pusilla, Vampyriscus brocki, Vampyriscus aff nymphaea y Vampyrodes caraccioli.

El estudio ha contribuido al incremento de especies para los bosques de colina baja en la cuenca alta del río Itaya con el reporte de 17 especies. Además, los registros de Vampyriscus aff. nymphaea, Vampyressa aff. melissa, Vampyressa aff. pusilla, y Tonatia aff. bidens pueden ampliar su rango de distribución en la selva nororiental del Perú. Aunque es necesario mayor muestra para confirmar su presencia.

El análisis de Simpson $(\lambda)$ mostró alta dominancia, el cual se evidencia en las abundancias de las especies frugívoras: $R$. pumilio, A. obscurus y C. brevicauda. Estas especies son las que dominan a nivel de estratos, donde existe una alta probabilidad de que dos individuos escogidos al azar pertenezcan a la misma especie (Figura 3).

La distribución de proporciones de murciélagos con relación a algunas variables como el tamaño, edad y gremio alimentario mostró un patrón bien marcado (Tabla 3). En cuanto al tamaño, encontramos mayor cantidad de murciélagos pequeños, seguido de los medianos. Los grandes estuvieron en menor proporción. Mientras que en el análisis etario, hubo mayor proporción de adultos, y en menor proporción los sub adultos y juveniles. Respecto a los gremios alimentarios, también se tuvo jerarquía de proporciones, siendo mayor los frugívoros, seguido de los insectívoros. Estas proporciones desiguales $(\mathrm{P}<0.05)$ muestran un mismo patrón en el sotobosque y medio-dosel $(\mathrm{P}<$ 0.0001).

En el bosque de colina baja se registró 47 especies, pero se estima una riqueza de 59 especies en la zona de estudio (Figura 4). Además, se evidenció una diferencia significativa entre la riqueza de especies observadas por estratos, siendo mayor en el sotobosque $(t=3.28, \mathrm{p}=0.01)$. En tanto, la diversidad verdadera fue de 24.54 especies efectivas, sin ninguna diferencia a nivel de estratos $(t=0.94, \mathrm{p}=0.24)$ (Tabla 2$)$. 


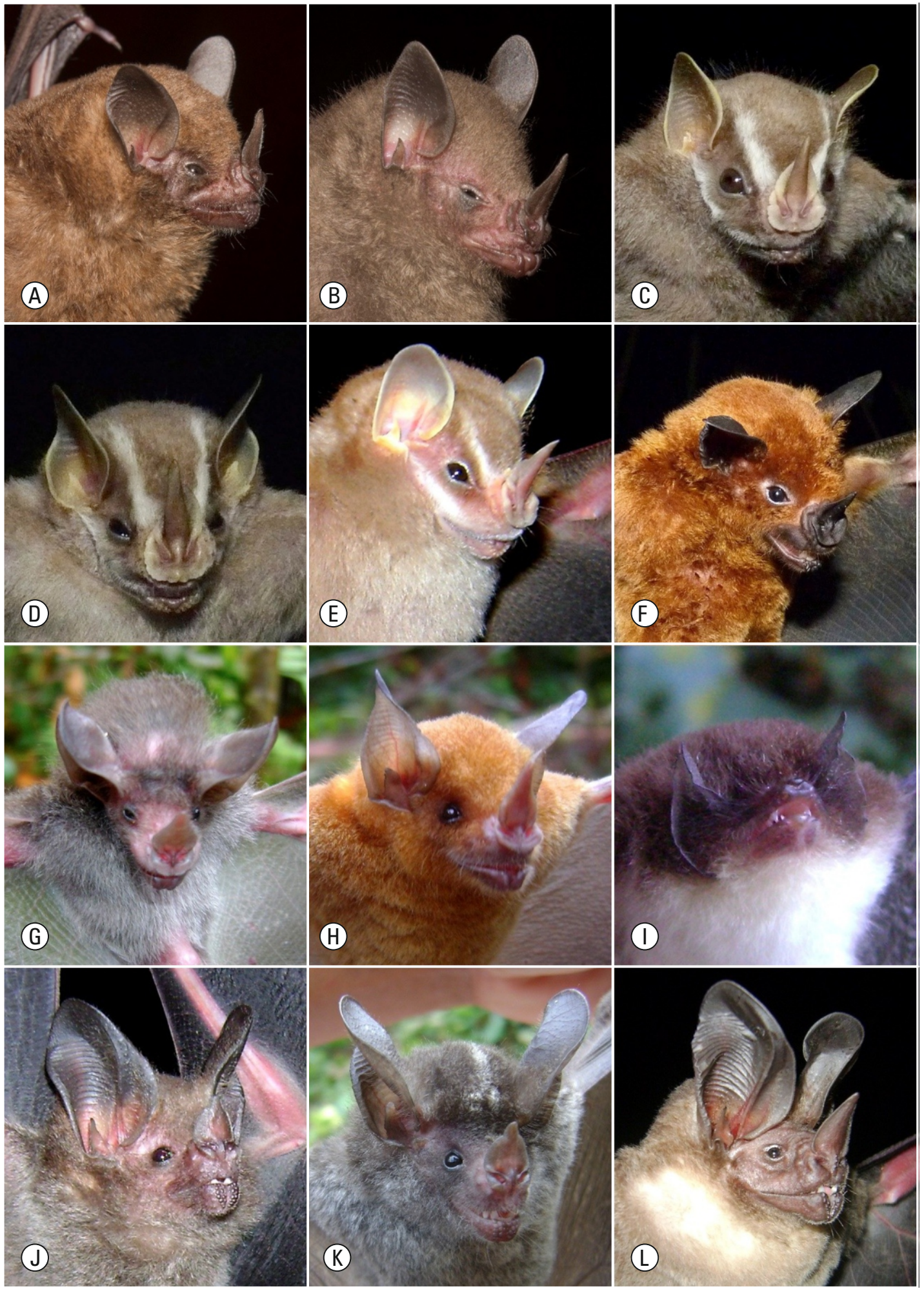

Figura 2. Algunos murciélagos capturados en bosques de colina de la cuenca alta del Itaya: A. Carollia benkeithi, B. Rhinophylla pumilio, C. Vampyriscus aff. nymphaea, D. Vampyressa aff. pusilla, E. Dermanura glauca. F. Phyllostomus hastatus. G. Micronycteris minuta, H. Micronycteris megalotis, I. Thyroptera tricolor, J. Tonatia aff. bidens K. Tonatia saurophyla, L. Lophostoma silvicola. 
Tabla 1. Riqueza de murciélagos por estratos en bosques de colina baja de la cuenca alta del Itaya.

\begin{tabular}{|c|c|c|c|c|c|}
\hline \multirow{2}{*}{$\begin{array}{l}\text { Familia } \\
\text { Sub Familia } \\
\text { Especie }\end{array}$} & \multicolumn{2}{|c|}{ Estrato Vertical } & \multirow{2}{*}{ Tamaño } & \multirow{2}{*}{$\begin{array}{c}\text { Gremio } \\
\text { Alimentario }\end{array}$} & \multirow{2}{*}{$\begin{array}{l}\text { Categoría } \\
\text { UICN }\end{array}$} \\
\hline & $\mathbf{S}$ & M D & & & \\
\hline \multicolumn{6}{|l|}{ EMBALLONURIDAE } \\
\hline Peropteryx macrotis & $x$ & & M & 1 & LC \\
\hline Saccopteryx bilineata & $x$ & $x$ & M & 1 & LC \\
\hline Saccopteryx leptura* & $\mathrm{x}$ & $x$ & $\mathrm{P}$ & 1 & LC \\
\hline \multicolumn{6}{|l|}{ PHYLLOSTOMIDAE } \\
\hline \multicolumn{6}{|l|}{ CAROLLIINAE } \\
\hline Carollia benkeithi* & $x$ & & $P$ & $\mathrm{~F}$ & LC \\
\hline Carollia brevicauda & $x$ & $x$ & $P$ & $\mathrm{~F}$ & LC \\
\hline Carollia perspicillata & $x$ & $x$ & $P$ & $\mathrm{~F}$ & LC \\
\hline \multicolumn{6}{|l|}{ DESMODONTINAE } \\
\hline Desmodus rotundus & $x$ & & M & $\mathrm{H}$ & LC \\
\hline \multicolumn{6}{|l|}{ GLOSSOPHAGINAE } \\
\hline Choeroniscus minor & $x$ & $x$ & & $\mathrm{~N}$ & LC \\
\hline Glossophaga commissarisi* & $x$ & & $P$ & $\mathrm{~N}$ & LC \\
\hline Glossophaga soricina* & $x$ & & $P$ & $\mathrm{~N}$ & LC \\
\hline Lichonycteris obscura* & $x$ & & $P$ & $\mathrm{~N}$ & LC \\
\hline \multicolumn{6}{|l|}{ GLYPHONYCTERINAE } \\
\hline Trinycteris nicefori* & & $x$ & $P$ & 1 & LC \\
\hline \multicolumn{6}{|l|}{ LONCHOPLYLLINAE } \\
\hline Hsunycteris thomasi* & $x$ & $x$ & $P$ & $\mathrm{~N}$ & LC \\
\hline \multicolumn{6}{|l|}{ MICRONYCTERINAE } \\
\hline Micronycteris megalotis & $x$ & $x$ & $P$ & I & LC \\
\hline Micronycteris minuta & $x$ & $x$ & $P$ & 1 & LC \\
\hline
\end{tabular}

PHYLLOSTOMINAE

$\begin{array}{llllll}\text { Lophostoma brasiliense* }^{*} & \text { X } & & \text { P } & \text { I } & \text { LC } \\ \text { Lophostoma carrikeri* }^{*} & & \text { X } & \text { M } & \text { I } & \text { LC } \\ \text { Lophostoma silvicola }_{\text {Mimon crenulatum }} & \text { X } & \text { X } & \text { M } & \text { I } & \text { LC } \\ \text { Phyllostomus elongatus } & \text { X } & \text { X } & \text { M } & \text { I } & \text { LC } \\ \text { Phyllostomus hastatus* }^{*} & \text { X } & \text { X } & \text { G } & \text { O } & \text { LC } \\ \text { Tonatia aff. bidens* }_{\text {Tonatia saurophila* }}^{*} & \text { X } & \text { X } & \text { M } & \text { I } & \text { DD } \\ \text { RHINOPHYLLINAE } & \text { X } & \text { X } & \text { M } & \text { I } & \text { LC } \\ \text { Rhinophylla fischerae } & \text { X } & \text { X } & \text { P } & \text { F } & \text { LC } \\ \text { Rhinophylla pumilio } & \text { X } & \text { X } & \text { P } & \text { F } & \text { LC }\end{array}$

STENODERMATINAE

$\begin{array}{llllll}\text { Artibeus lituratus } & \text { X } & \text { X } & \text { G } & \text { F } & \text { LC } \\ \text { Artibeus obscurus } & \text { X } & \text { X } & \text { M } & \text { F } & \text { LC } \\ \text { Artibeus planirostris } & \text { X } & \text { X } & \text { G } & \text { F } & \text { LC }\end{array}$




\begin{tabular}{|c|c|c|c|c|c|}
\hline \multirow{2}{*}{$\begin{array}{l}\text { Familia } \\
\text { Sub Familia } \\
\text { Especie }\end{array}$} & \multicolumn{2}{|c|}{ Estrato Vertical } & \multirow{2}{*}{ Tamaño } & \multirow{2}{*}{$\begin{array}{c}\text { Gremio } \\
\text { Alimentario }\end{array}$} & \multirow{2}{*}{$\begin{array}{l}\text { Categorí́ } \\
\text { UICN }\end{array}$} \\
\hline & $\mathbf{S}$ & M D & & & \\
\hline Chiroderma villosum & $x$ & $x$ & $M$ & $\mathrm{~F}$ & LC \\
\hline Dermanura anderseni & $x$ & & $P$ & $\mathrm{~F}$ & LC \\
\hline Dermanura glauca & $x$ & $x$ & $P$ & $\mathrm{~F}$ & LC \\
\hline Dermanura gnoma & $x$ & $x$ & $P$ & $\mathrm{~F}$ & LC \\
\hline Mesophylla macconnelli & $x$ & $x$ & $P$ & $\mathrm{~F}$ & LC \\
\hline Sturnira lilium & $x$ & $x$ & $P$ & $\mathrm{~F}$ & LC \\
\hline Sturnira magna & & $x$ & $M$ & $\mathrm{~F}$ & LC \\
\hline Sturnira tildae & $x$ & $x$ & M & $\mathrm{F}$ & LC \\
\hline Uroderma bilobatum & & $x$ & $P$ & $\mathrm{~F}$ & LC \\
\hline Uroderma magnirostrum & $x$ & $x$ & $P$ & $\mathrm{~F}$ & LC \\
\hline Vampyressa aff. melissa* & $x$ & & $P$ & $\mathrm{~F}$ & VU \\
\hline Vampyressa aff. pusilla* & $x$ & & $P$ & $\mathrm{~F}$ & $\mathrm{DD}$ \\
\hline Vampyriscus bidens & $x$ & $x$ & $P$ & $\mathrm{~F}$ & LC \\
\hline Vampyriscus brocki & & $x$ & $P$ & $\mathrm{~F}$ & LC \\
\hline Vampyriscus aff. nymphaea* & & $x$ & $P$ & $\mathrm{~F}$ & LC \\
\hline Vampyrodes caraccioli* & & $x$ & $\mathrm{M}$ & $\mathrm{F}$ & $\mathrm{LC}$ \\
\hline \multicolumn{6}{|l|}{ THYROPTERIDAE } \\
\hline Thyroptera tricolor* & & $x$ & $\mathrm{P}$ & $\mathrm{I}$ & $\mathrm{LC}$ \\
\hline \multicolumn{6}{|l|}{ VESPERTILIONIDAE } \\
\hline Myotis nigricans & $\mathrm{x}$ & $x$ & $P$ & 1 & LC \\
\hline Myotis riparius* & $x$ & $x$ & $P$ & I & LC \\
\hline
\end{tabular}

\section{DISCUSIÓN}

La riqueza de especies registrada en este estudio (47 especies) superó ampliamente al estudio de Calderón \& Rengifo (2009), quienes aplicando las técnicas de búsqueda de refugios y redes de neblina y un esfuerzo de 1560 horas/red registraron 30 especies. Nuestros registros fueron también superior al de Cevillano \& Ramos (2011) quienes emplearon un esfuerzo de 1296 horas/red y registraron solamente 20 especies frugívoras. La diferencia marcada entre las cantidades de especies encontradas entre los estudios mencionados, fue probablemente debido a la ubicación de redes de neblina a nivel de estratos, el cual permitió capturas exclusivas en el estrato superior del bosque, como Lophostoma carrikeri (1 ind.), Thyroptera tricolor (1 ind.), Trinycteris nicefori (5 ind.), V. brocki (1 ind.) y Vampyrodes caraccioli (1 ind.).
Algunos estudios indican que las tres primeras especies insectívoras son exclusivas de sotobosque (Bernand, 2001; Camacho et al., 2008 y Fonseca \& Pinto, 2004), quizá sus conclusiones sesgadas se deben al diseño de muestreo. También, probablemente la distribución vertical de estas especies en bosque de colina difiere con otros bosques de tierra firme o podrían corresponder a capturas ocasionales en medio dosel, a excepción de Trinycteris nicefori, el cual fue capturado 5 veces en el medio dosel, mostrando exclusividad por este estrato en bosques de colina. Otros factores que pueden haber influenciado positivamente en el estudio son: el tipo de hábitat, esfuerzo y estación de muestreo. Sin embargo, nuestros registros fueron inferiores a estudios como: Medina et al. (2015), quienes aplicando un esfuerzo de 284 redes/noche lograron registrar 52 especies. También resultó 


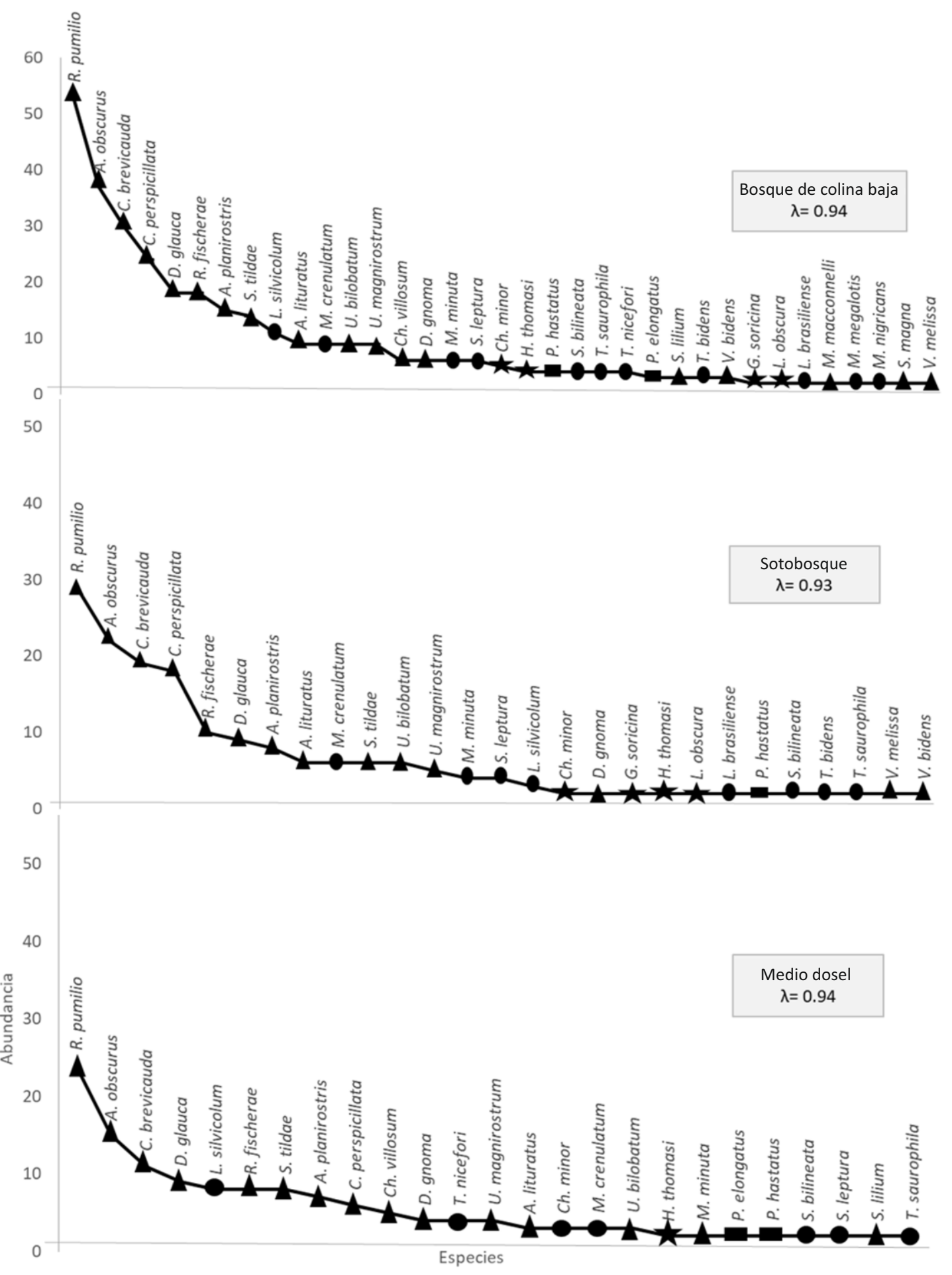

Figura 3. Dominancia de especies por estrato vertical en bosque de colina baja. Los símbolos representan los tipos de gremio alimenticio (Frugívoros = triangulo, Insectívoros = circulo, Nectarívoros = estrella, Omnívoros $=$ rectángulo). 
inferior al de Arias (2008), Hice et al. (2004) y Ascorra et al. (1993) quienes aplicando esfuerzos superiores a 2257 horas/red, registraron 56, 63 y 62 especies para el ámbito de la Carretera Iquitos Nauta, Reserva Nacional Allpahuayo Mishana (RNAM) y cuenca del río Ucayali; la riqueza de especies en estos estudios está influenciado por el esfuerzo de captura superior a nuestro estudio.

Comparando la diversidad de especies registradas entre el sotobosque y medio dosel, encontramos diferencias significativas en la riqueza pero no en la diversidad verdadera. Estos resultados podrían estar influenciados por la altura muestreada, ya que es posible que exista un grupo de especies no conocidas que frecuentan alturas mayores a $16 \mathrm{~m}$, nuestro límite superior, como ocurrió con los registros en bosques primarios de Brasil (Bernard, 2001), donde se encontró diferencias en la diversidad de especies entre dosel $(17-30 \mathrm{~m})$ y sotobosque $(0-2.5 \mathrm{~m})$. Así también en bosques de Asia y África (Francis, 1994; Henry et al., 2004) se registró mayor diversidad y abundancia de murciélagos en el dosel, entre los 30 y $55 \mathrm{~m}$ de altura. Estos tipos de estudios aún faltan realizar en la Amazonía peruana, seguramente deben existir muchas especies nuevas que frecuentan estratos superior a los $16 \mathrm{~m}$ de altura.

En el estudio se reporta cuatro especies aún no confirmadas: Vampyriscus aff. nymphaea, Vampyressa aff. melissa, Vampyressa aff. pusilla y Tonatia aff. bidens, el cual indicaría una ampliación de su rango de distribución en la Amazonia, por ello, sería de gran interés implementar métodos integrados de campo y laboratorio con análisis de secuenciación del ADN, para así corroborar la distribución de estas especies en el Perú.

En cuanto a la diversidad, obtuvimos en promedio 25 especies efectivas de los bosques de colina baja. El $26 \%$ de especies capturadas probablemente corresponden a raras u ocasionales que requieren de un mayor esfuerzo para ser capturados, o quizá corresponden a especies que frecuentan los estratos de dosel y emergente superiores a los $16 \mathrm{~m}$ que podrían ser identificados con otros métodos como la bioacústica, identificación de refugios o combinación de métodos. Al respecto, Calderon \& Rengifo (2009) mencionan siete especies que no fueron registrados en nuestro estudio (Peropteryx leucoptera, Rhynchonycteris naso, Lichonycteris degener, Lonchophylla robusta, Trachops cirrhosus, Plathyrrinus incarum, Furipterus horren), las mismas que resultan de la aplicación de la combinación de método por redes de neblina y refugios, incrementando a un $92 \%$ la riqueza de especies para la cuenca alta del río Itaya.
Las especies que dominan los bosques de colina baja son principalmente frugívoras, entre ellas $R$. pumilio, A. obscurus y C. brevicauda. De ellas, las dos primeras han sido denominadas como principales agentes dispersores en estos bosques, alimentándose principalmente de frutos de los géneros Philodendron, Asplundia, Ficus, Cecropia, Marcgravia, Piper, entre otros (Cevillano \& Ramos, 2011). La dominancia de algunos grupos de murciélagos son indicadores de los estados del ecosistema, como las especies de la familia Phyllostomidae (Fenton et al., 1992; Kalko et al., 1996) y su amplia gama de tendencias alimenticias como frugívoros, insectívoros, carnívoros, nectarívoros, hematófagos (Baker et al., 1976) e incluso omnívoros. El número de especies o de hábitos alimentarios hace caracterizarlos como indicadores del estado de alteración del hábitat principalmente por actividades humanas (Fenton et al., 1992; Wilson et al., 1996; Medellín et al., 2000).

Los murciélagos presentan un patrón bien marcado en la distribución vertical por tamaño, edad y gremio alimentario, mostrando el mismo patrón en los diferentes estratos, esto nos permite mencionar que los murciélagos de tamaños pequeño, de edad adulto $\mathrm{y}$ de gremio frugívoro representan las especies efectivas en bosques de colina.

En la cuenca alta del río Itaya, los bosques de colina presentan una composición de especies de murciélagos que indican que el estado del bosque aún es saludable, en donde dominan las especies frugívoras que se alimentan de especies esciófitas como algunas pioneras y otras de estadios de bosques maduros (Cevillano \& Ramos, 2011), donde la formación de claros permite el establecimiento de especies dispersadas por murciélagos. Además, la frecuencia de murciélagos insectívoros, de tamaño grande, valida la clasificación de calidad de un bosque (Feton, 1992). Sin embargo, consideramos que para un mayor entendimiento de las condiciones de calidad de un ecosistema, es ideal además del conocimiento de la diversidad de especies, la composición de la dieta. Por ejemplo, en zonas alteradas dominan especies frugívoras, cuya composición de su dieta son básicamente especies pioneras, como las Piperaceae, Solanaceae, Urticaceae, Melastomataceae (Gorchov et al., 1993; Gonzales, 1998; Olea-Wagner et al., 2007; Mena, 2010) en este mismo tipo de bosque no existen o hay escasos registros de especies insectívoras de la sub Familia Phyllostominae (Fenton et al., 1992; Brosset et al., 1996; Kalko et al., 1996). El cual no ocurre en estos bosques por ser de moderada y moderada-alterada perturbación según la referencia de Aquino et al. (2012). La especies de los géneros Micronycteris y 
Tonatia poseen hábitos alimentarios y comportamiento muy especializados en recoger insectos de las plantas y superficie del suelo, en ambientes muy bien conservados donde abundan escarabajos, saltamontes, mariposas nocturnas y polillas; sin embargo, los componentes de estos géneros son muy sensibles y desaparecen en lugares con alta perturbación de hábitat (Kalko et al.,1996). En este estudio reportamos la presencia de Micronycteris megalotis, Micronycteris minuta, Tonatia saurophila, Tonatia aff bidens, las cuales indican que los bosques en el área de estudio todavía se mantiene en buen estado de conservación.

Tabla 2. Índice de diversidad de murciélagos en bosque de colina y estratos de sotobosque y medio dosel.

\begin{tabular}{lcccccc}
\hline Índice de Diversidad & $\begin{array}{c}\text { Bosque de } \\
\text { Colina }\end{array}$ & Sotobosque & Medio Dosel & \multicolumn{2}{c}{$\begin{array}{c}\text { Comparaciones } \\
\text { por Estrato }\end{array}$} \\
\cline { 5 - 7 } & & & & & ${ }^{0} \mathbf{D}$ & ${ }^{1} \mathbf{D}$ \\
\hline Riqueza de especies ('D) & 47 & 41 & 36 & & \\
\hline$N^{\circ}$ de individuos & 345 & 193 & 152 & $\mathrm{t}=3.28, \quad \mathrm{t}=0.94$ \\
\hline Simpson_1-D $(\lambda)$ & 0.94 & 0.9322 & 0.938 & $\mathrm{p}=0.01$ & $\mathrm{p}=0.24$ \\
\hline Shannon_H & 3.20 & 3.112 & 3.14 & & \\
\hline Diversidad verdadera ('D) & 24.54 & 22.47 & 23.1 & & \\
\hline
\end{tabular}
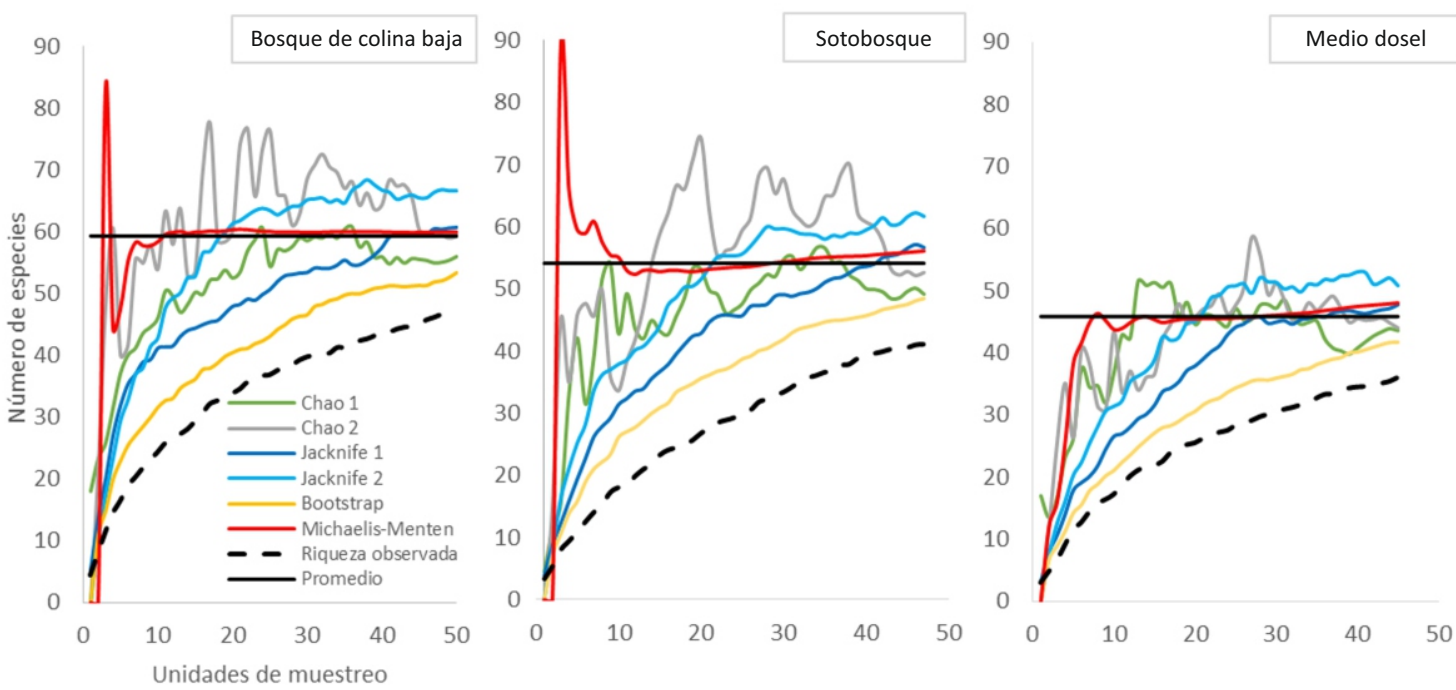

Figura 4. Riqueza esperada por estimadores no paramétricos en bosque de colina baja a nivel de estrato vertical del bosque. 
Tabla 3. Homogeneidad de proporciones de murciélagos por estrato vertical (sotobosque y medio-dosel) en relación a las variables de tamaño, edad y gremio alimentario.

\begin{tabular}{|c|c|c|c|c|c|}
\hline & & \multicolumn{4}{|c|}{ Estrato vertical } \\
\hline \multicolumn{2}{|c|}{ Variables } & \multicolumn{2}{|c|}{$N^{\circ}$ Individuos } & \multirow{2}{*}{ G -test } & \multirow{2}{*}{$\mathbf{P}$} \\
\hline & & Sotobosque & Medio dosel & & \\
\hline \multirow{3}{*}{ Tamaño } & Grande & 17 & 14 & \multirow{3}{*}{166.23} & \multirow{3}{*}{$<0.0001$} \\
\hline & Mediano & 47 & 47 & & \\
\hline & Pequeño & 129 & 91 & & \\
\hline G-test/p & & $104.74 /<0001$ & $63.50 /<0001$ & & \\
\hline \multirow{3}{*}{ Edad } & Adulto & 3 & 3 & \multirow{3}{*}{303.61} & \multirow{3}{*}{$<0.0001$} \\
\hline & Sub adulto & 43 & 47 & & \\
\hline & Juvenil & 146 & 102 & & \\
\hline G-test/p & & $188.26 /<0001$ & $118.72 /<0001$ & & \\
\hline \multirow{4}{*}{$\begin{array}{l}\text { Gremio } \\
\text { Alimentario }\end{array}$} & Frugívoro & 151 & 115 & \multirow{4}{*}{463.83} & \multirow{4}{*}{$<0.0001$} \\
\hline & Insectívoro & 28 & 28 & & \\
\hline & Nectarívoro & 9 & 5 & & \\
\hline & Omnívoro & 4 & 4 & & \\
\hline G-test/p & & $165.92 /<0001$ & $199.30 /<0001$ & & \\
\hline
\end{tabular}

\section{CONCLUSIONES}

Este estudio evidencia una gran diversidad de especies de murciélagos para los bosques de colina, con una riqueza de 47 especies y una diversidad verdadera de 24.51 especies efectivas, donde los murciélagos frugívoros dominan y los insectívoros resultan frecuentes, evidenciando el estado saludable del bosque según sus características indicadoras de perturbación de hábitat. La riqueza resultó mayor en estrato inferior y la diversidad verdadera fue similar entre los estratos. Las proporciones de tamaño, edad y gremio alimentario se mantienen en los dos estratos. En tanto el 26\% de especies capturadas corresponden a únicos registros, siendo éstas las más susceptibles ante perturbaciones antropogénicas que podrían ocasionar extinciones locales.

\section{AGRADECIMIENTOS}

Con profusa consideración a la Señorita Aura Murrieta de la ONG ACEER y a la entidad del IIAP Iquitos, quienes financiaron el proyecto. A nuestros guías de campo: Royler, Eduardo, Dixner y Nelly quienes nos ayudaron con el sistema de polea con cuerdas para ubicar las redes de neblina en estrato medio-dosel así como su acompañamiento durante los muestreos en campo.

\section{REFERENCIAS BIBLIOGRÁFICAS}

Aquino, R.; Ramos, M.C.; Charpentier, E.; García, M. 2012. Fauna silvestre, Proyecto Microzonificación Ecológica y Económica del Área de Influencia de la Carretera IquitosNauta. IIAP y DEVIDA. Iquitos. 86pp.

Aquino, R.; Gil, D.; Pezo, E. 2009. Aspectos ecológicos y sostenibilidad de la caza del majás (Cuniculus paca) en la cuenca del río Itaya, Amazonía peruana. Revista Peruana de Biología, 16(1): 67-72.

Arias, L.C. 2008. Ecomorphological structure of an Amazonian Phyllostomid bat assemblage. Tesis de Maestría, Texas Tech University, United States of America. 103pp.

Ascorra, C.F.; Gorchov, D.L.; Cornejo, F. 1993. The bats from Jenaro Herrera, Loreto, Peru. Mammalia, 57(4): 533-552.

Ayres, M.; Ayres, M.Jr.; Ayres, D.L.; Santos, A.S. 2007. BioEstat 5.0. Aplicações Estatísticas nas Áreas das Ciências BioMédicas. Sociedade Civil de Mamirauá, Belém. 323pp.

Baker, R.J.; Jones, J.K.; Carter, D.C. 1976. Biology of bats of the New World Family Phyllostomatidae. Part I. Spec. Publ. Mus., Texas Tech Univ., 10:1-218. 
Baker, R.J.; McDonough, M.M.; Swier, V.J.; Larsen, P.A.; Carrera, J.P.; Ammerman, L.K. 2009. New species of bonneted bat, genus Eumops (Chiroptera: Molossidae) from the lowlands of western Ecuador and Peru. Acta Chiropterologica, 11(1): 1-13.

Bernard, E. 2001. Vertical stratification of bat communities in primary forests of Central Amazon, Brazil. Journal of Tropical Ecology, 17(1): 115-126.

Bravo, A. 2009. Collpas as activity hotspots for frugivorous bats (Stenodermatinae) in the Peruvian Amazon: Underlying mechanisms and conservation implications. Tesis Doctoral. Universidad Agraria la Molina, Lima. 140pp.

Brosset, A.; Charles-Dominique, P.; Cockle, A.; Cosson, J.F.; Masson, D. 1996. Bat communities and deforestation in French Guiana. Canadian Journal of Zoology, 74(11): 1974-1982.

Calderon, W.; Pacheco, V. 2012. First report of Artibeus bogotensis Andersen, 1906 (Chiroptera: Phyllostomidae) for Peru. Check List, 8: 1333-1336.

Calderón, W.L.; Rengifo, E.M. 2010. Diversidad y uso de hábitat por micromamíferos en la cuenca alta del río Itaya. Tesis de pre grado. Universidad Nacional de la Amazonia Peruana, Facultad de Ciencias Biológicas, Iquitos. 74pp.

Camacho, M. A.; Tirira, D. G.; Dick, C. W.; Burneo, S. F. 2014. Lophostoma carrikeri (Allen, 1910) (Chiroptera: Phyllostomidae): first confirmed records in Ecuador. Check List, 10(1), 217-220.

Cevillano, S.C; Ramos, M.C. 2011. Nicho alimentario de murciélagos frugívoros en bosque de colina, río Itaya, Loreto-Perú. Tesis de pre grado. Universidad Nacional de la Amazonia Peruana, Facultad de Ciencias Biológicas, Iquitos. $116 \mathrm{pp}$.

Díaz, M.M. 2011. New records of bats from the northern region of the Peruvian Amazon. Zoological Research, 32(2): 168-178.

Díaz, M.M.; Solari, S.; Aguirre, L.F.; Aguiar, L.M.; Barquez, R.M. 2016. Clave de Identificación de los Murciélagos de Sudamérica-Chave de Identificacao dos Morcegos da America do Sul. Publicación Especial Número 2.97 pp.

Encarnación, F. 1993. El bosque y las formaciones vegetales en la llanura amazónica del Perú. Alma Mater, 6: 95-114.

Fenton, M.B.; Acharya, L.; Audet, D.; Hickey, M.B.C.; Merriman, C.; Obrist, M.K.; Syme, D.M.; Adkins, B. 1992. Phyllostomid bats (Chiroptera: Phyllostomidae) as indicators of habitat disruption in the Neotropics. Biotropica 24(3): 440-446.
Fernández, G.J.; Torres, M.I. 2013. Lista actualizada de quirópteros de los Departamentos de Loreto Ucayali y Madre de Dios (Perú). Barbastella, 6(1): 73-88.

Fonseca, R.; Pinto, M. 2004. A new Lophostoma (Chiroptera: Phyllostomidae:

Francis, C.M. 1994. Vertical stratification of fruit bats (Pteropodidae) in lowland dipterocarp rainforest in Malaysia. Journal of Tropical Ecology, 10(4): 523-530.

García-Morales, R.; Moreno, C.E.; Bello-Gutiérrez, J. 2011. Renovando las medidas para evaluar la diversidad en comunidades ecológicas: el número de especies efectivas de murciélagos en el sureste de Tabasco, México. Therya, 2(3): 205-215.

Gardner, A.L. (Ed.) 2008. Mammals of South America, volume 1: marsupials, xenarthrans, shrews, and bats (Vol. 1). University of Chicago Press. p. $187-481$.

González, J.G. 1998. Dispersión de semillas por murciélagos: su importancia en la conservación y regeneración del bosque tropical. Acta Zoológica Mexicana (nueva serie), 73: 57-74.

Gorchov, D.L.; Cornejo, F.; Ascorra, C.; Jaramillo, M. 1993. The role of seed dispersal in the natural regeneration of rain forest after strip-cutting in the Peruvian Amazon. Vegetatio, 107(1): 339349.

Henry, M.; Barriere, P.; Gautier-Hion, A.; Colyn, M. 2004. Species composition, abundance and vertical stratification of a bat community (Megachiroptera: Pteropodidae) in a West African rain forest. Journal of Tropical Ecology, 20(1): 21-29.

Hice, C.L.; Velazco, P.M.; Willig, M.R. 2004. Bats of the Reserva Nacional Allpahuayo-Mishana, northeastern Peru, with notes on community structure. Acta Chiropterologica, 6(2):319-334.

Hill, M.O. 1973 Diversity and evenness: a unifying notation and its consequences. Ecology, 54(2):427-432.Hammer, Ø.; Harper, D.A.T.; Ryan, P.D. 2001. PAST-Palaeontological statistics.( http://folk.uio.no/ohammer/past/). Acceso 05/10/2017.

Jones, C.; McShea, W.J.; Conroy, M.J.; Kunz, T.H. 1996. Capturing Mammals. In: Wilson, D.F.; Cole, F.R.; Nichols, J.D.; Rudran, R.; Foster, M.S. (ed). Measuring and monitoring biological diversity: Standard Methods for Mammals. Washington, D.C.: Smithsonian Institution Press. p.115- 155.

Jost, L. 2006. Entropy and diversity. Oikos, 113(2): 363-375. 
Kalko, E.K.; Herre, E.A.; Handley, C.O. 1996. Relation of fig fruit characteristics to fruit-eating bats in the New and Old World tropics. Journal of Biogeography, 23(4): 565-576.

Kalko, E.K.; Herre, E.A.; Handley, C.O. 1996. Relation of fig fruit characteristics to fruit-eating bats in the New and Old World tropics. Journal of Biogeography, 23(4): 565-576.

Kunz, T.H.; Kurta, A. 1988. Capture methods and holding devices. In: Kunz, T.H.(Ed.). Ecological and behavioral methods for the study of bats. $\mathrm{p}$. 1-29.

Lim, B.K.; Engstrom, M.D.; Reid, F.A.; Simmons, N.B.; Voss, R.S.; Fleck, D.W. 2010. A new species of Peropteryx (Chiroptera: Emballonuridae) from western Amazonia with comments on phylogenetic relationships within the genus. American Museum Novitates, 3686: 120.

Lopez-Baucells, A.; Rocha, R.; Bobrowiec, P.E.D.; Palmeirim, J.M.; Meyer, C.F.J. 2016. Field guide to Amazonian bats. Manaus. 174pp.

Maclaurin, J.; Sterelny, K. 2008. What is biodiversity? University of Chicago Press, Chicago. 217pp.

Medellín, R.A., Equihua, M.; Almin, M.A. 2000. Bat diversity and abundance as indicators in Neotropical forests. Conservation Biology, 14:1666-1675.

Medina, C.E.; López, E.; Pino, K.; Pari, A.; Zeballos, H. 2015. Biodiversidad de la zona reservada Sierra del Divisor (Perú): una visión desde los mamíferos pequeños. Revista peruana de biología, 22(2): 199-212.

Mena, J.L. 2010. Respuestas de los murciélagos a la fragmentación del bosque en Pozuzo, Perú. Revista Peruana de Biología, 17(3): 277-284.

MINAM 2015. Inventario y evaluación de los bosques de las cuencas de los ríos Itaya, Nanay y Tahuayo. Dirección General de Evaluación, Valoración y Financiamiento del Patrimonio Natural, Lima. 136pp.

Moreno, C.E.; Barragán, F.; Pineda, E.; Pavón, N.P. 2011. Reanálisis de la diversidad alfa: alternativas para interpretar y comparar información sobre comunidades ecológicas. Revista mexicana de biodiversidad, 82(4): 12491261.

Muñoz, J. 2001. Los murciélagos de Colombia. Sistemática, distribución, descripción, historia natural y ecológica. Editorial Universidad de Antioquia. Antioquia. 391pp.
Nagorsen, D.W.; Peterson, R.L. 1980. Mammal collector's manual: a guide for collecting, documenting, and preparing mammal specimens for scientific research. Royal Ontario Museum, Toronto. 79pp.

Olea-Wagner, A.; Lorenzo, C.; Naranjo, E.; Ortiz, D.; León-Paniagua, L. 2007. Diversidad de frutos que consumen tres especies de murciélagos (Chiroptera: Phyllostomidae) en la selva lacandona, Chiapas, México. Revista mexicana de biodiversidad, 78(1): 191-200.

Paredes, M. 2012. Clima, Documento Temático. Proyecto Microzonificación Ecológica y Económica del Área de Influencia de la Carretera Iquitos-Nauta. IIAP y DEVIDA. Iquitos. 88pp.

Fonseca, R.M.; Pinto, C.M. 2004. A new Lophostoma (Chiroptera: Phyllostomidae: Phyllostominae) from the Amazonia of Ecuador. Occasional Papers 242: 1-9.

Quintana, H.; Pacheco, V.; Salas, E. 2009. Diversidad y conservación de los mamíferos de Ucayali, Perú. Ecología Aplicada, 8(1-2): 91103.

Seaby, R.M.H.; Henderson P.A. 2007. Species diversity and richness version 4.1.2. PISCES Conservation Limited. (http://www.piscesconservation.com/freestuff.html). Acceso 15/09/2017.

Simmons, N. B. 2006. Order Chiroptera. In Wilson, D.E.; Reeder, D.M. eds. Mammal species of the world: a taxonomic and geographic reference. 3 rd ed. Smithsonian Institution Press, Washington, DC. p. 312-529.

Solari, S.; Martínez-Arias, V. 2014. Cambios recientes en la sistemática y taxonomía de murciélagos Neotropicales (Mammalia: Chiroptera). Therya, 5(1): 167-196.

Tirira, D. 1999. Mamíferos del Ecuador (Vol. 2). Museo de Zoología, Centro de Biodiversidad y Ambiente, Pontificia Universidad Católica del Ecuador. Quito. 392 pp.

Velazco, P.M. 2017. Murciélagos del Perú. (http://www.paulvelazco.com/murcielagos_per u.html). Acceso: 25/09/2017.

Velazco, P.M., Gregorin, R.; Voss, R.S.; Simmons, N.B. 2014. Extraordinary local diversity of diskwinged bats (Thyropteridae: Thyroptera) in northeastern Peru, with the description of a new species and comments on roosting behavior. American Museum Novitates, 3795: 1-28.

Velazco, P.M.; Gardner, A.L.; Patterson, B.D. 2010. Systematics of the Platyrrhinus helleri species complex (Chiroptera: Phyllostomidae), with descriptions of two new species. Zoological Journal of the Linnean Society, 159(3): 785-812. 
Voss, R.S.; Emmons, L. 1996. Mammalian diversity in Neotropical lowland rainforests: a preliminary assessment. Bulletin of the AMNH.18-22.

Wilson, D. E., Ascorra, C. F., \& Solari, S. 1996. Bats as indicators of habitat disturbance. In D.E. Wilson, D.E.; Sandoval, A. (Eds). Manu, the Biodiversity of Southeastern Peru. Manu: the biodiversity of southeastern Peru. p. 613-625.
Zárate, R.; Mori, T.J.; Maco-García, J.T. 2013. Estructura y composición florística de las comunidades vegetales del ámbito de la carretera Iquitos-Nauta, Loreto-Perú. Folia Amazónica, 22(1-2): 77-89.

Recibido: 24 de Julio del 2017

Aceptado para publicación: 11 de Agosto del 2017 